\title{
Using an integrative machine learning approach utilising homology modelling to clinically interpret genetic variants: CACNA1F as an exemplar
}

\author{
Shalaw R. Sallah $\mathbb{B}^{1,2} \cdot$ Panagiotis I. Sergouniotis $\mathbb{1}^{2} \cdot$ Stephanie Barton $^{2} \cdot$ Simon Ramsden $^{2} \cdot$ Rachel L. Taylor $\mathbb{B}^{2} \cdot$ \\ Amro Safadi $^{1} \cdot$ Mitra Kabir $^{1} \cdot$ Jamie M. Ellingford ${ }^{2} \cdot$ Nick Lench $^{3} \cdot$ Simon C. Lovell $^{1} \cdot$ Graeme C. M. Black $^{1,2}$
}

Received: 13 May 2019 / Revised: 13 January 2020 / Accepted: 10 March 2020 / Published online: 20 April 2020

(c) The Author(s) 2020. This article is published with open access

\begin{abstract}
Advances in DNA sequencing technologies have revolutionised rare disease diagnostics and have led to a dramatic increase in the volume of available genomic data. A key challenge that needs to be overcome to realise the full potential of these technologies is that of precisely predicting the effect of genetic variants on molecular and organismal phenotypes. Notably, despite recent progress, there is still a lack of robust in silico tools that accurately assign clinical significance to variants. Genetic alterations in the CACNA1F gene are the commonest cause of X-linked incomplete Congenital Stationary Night Blindness (iCSNB), a condition associated with non-progressive visual impairment. We combined genetic and homology modelling data to produce CACNA1F-vp, an in silico model that differentiates disease-implicated from benign missense $C A C N A 1 F$ changes. CACNA1F-vp predicts variant effects on the structure of the CACNAIF encoded protein (a calcium channel) using parameters based upon changes in amino acid properties; these include size, charge, hydrophobicity, and position. The model produces an overall score for each variant that can be used to predict its pathogenicity. CACNA1F-vp outperformed four other tools in identifying disease-implicated variants (area under receiver operating characteristic and precision recall curves $=0.84$; Matthews correlation coefficient $=0.52$ ) using a tenfold cross-validation technique. We consider this protein-specific model to be a robust stand-alone diagnostic classifier that could be replicated in other proteins and could enable precise and timely diagnosis.
\end{abstract}

Supplementary information The online version of this article (https:// doi.org/10.1038/s41431-020-0623-y) contains supplementary material, which is available to authorized users.

Shalaw R. Sallah

Graeme.black@manchester.ac.uk

1 Division of Evolution and Genomic Sciences, School of Biological Sciences, Faculty of Biology, Medicines and Health, University of Manchester, Manchester Academic Health Science Centre, Manchester, UK

2 Manchester Centre for Genomic Medicine, Central Manchester University Hospitals NHS Foundation Trust, Manchester Academic Health Sciences Centre, St Mary's Hospital, Manchester, UK

3 Congenica Ltd, Biodata Innovation Centre, Wellcome Genome Campus, Hinxton, Cambridge, UK

\section{Introduction}

Over the past decade, high-throughput DNA sequencing technologies have revolutionised the management of individuals with rare genetic disorders, enabling timely and precise diagnosis, and facilitating personalised medicine approaches [1]. For genetically heterogenous conditions such as hereditary hearing loss and inherited retinal disorders (IRDs), genomic testing has been shown to have significant clinical utility, leading to improved management [2]. In these conditions, variant detection can provide a molecular diagnosis in over $50 \%$ of patients $[3,4]$. However, distinguishing the disease-causing variants from the many potentially functional variants present in any human genome remains particularly challenging [5].

IRDs is a heterogeneous group of disorders that affect $\sim 1$ in 3000 people [6] and are a leading cause of blindness in working age adults in the UK [7]. Congenital stationary night blindness (CSNB; also known as congenital stationary synaptic dysfunction/disorder) is a non-progressive form of 
childhood-onset IRD that is associated with variable combinations of night vision problems, reduced visual acuity, myopia, and nystagmus. X-linked incomplete CSNB (iCSNB; also known as type 2 CSNB (OMIM 300071)) is the most prevalent CSNB subtype and it is classically caused by variants in the CACNAIF gene [8]. CACNAIF (Gene ID 300110) consists of 48 exons (ENST00000376265.2) and encodes a polypeptide (1977 amino acids) that forms the pore of a voltage-gated calcium channel, $\mathrm{Ca}_{\mathrm{v}} 1.4 \alpha 1[9,10]$. CACNAIF (NM_005183.3). Its function involves sustaining continuous calcium dependent glutamate release from the photoreceptors to bipolar cells in the retina [11]. Over fifty $C A C N A 1 F$ missense variants are described to cause iCSNB on the human gene mutation database (HGMD v2019.4) [12] the majority of which are functionally uncharacterised. Improved prediction of the likely phenotypic consequences of missense variants in $C A C N A I F$ is therefore key to the molecular diagnosis of patients with iCSNB.

A number of in silico tools can be used for interpreting the effects of sequence variants, both in research and in clinical laboratory settings. Four commonly used tools include Sorting Intolerant From Tolerant (SIFT, [13]) which uses a sequence homology-based method, polymorphism phenotyping v2 (PolyPhen2, [14]) which utilises a sequence and structure-based approach, combined annotation dependent depletion (CADD, [15]) which uses a supervised learning method, and consensus deleteriousness score (CONDEL, [16]) which uses a consensus-based approach [17]. The latest version of CONDEL combines the predictions of two other tools, mutation assessor [18] and the Functional Analysis Through Hidden Markov Models ([19]) using weighted averaging.

Despite relative success in differentiating between disease-causing and benign variants in some genes, these tools are not consistently effective in their predictions [20]. Even in combination, their efficiency has been shown to be gene-dependent [21]. Here, we integrate detailed genetic $C A C N A 1 F$ data with homology modelling of the $\mathrm{Ca}_{\mathrm{v}} 1.4 \alpha 1$ protein structure. We show, using CACNA1F-variant predictor (CACNA1F-vp), that protein-specific structural analysis has the ability to improve performance in differentiating disease-implicated missense changes from other potentially functional variants.

\section{Methods}

\section{Datasets}

The $\mathrm{HGMD}^{\mathrm{R}}$ database was used to retrieve missense variants that have been previously associated with clinical phenotypes ( $n=63$; database accessed October 2017). The ClinVar database [22] was also interrogated and a literature search, using the search term 'CACNA1F AND mutation*', was carried out at the same time; no further changes were identified. DNA changes associated with disease in patients tested at the Manchester Genomic Diagnostic Laboratory (MGDL), a United Kingdom Accreditation Service Clinical Pathology Accredited medical laboratory (Clinical Pathology Accredited identifier, no. 4015) were also included ( $n$ =9; database accessed October 2017). The guidelines set out by the American College of Medical Genetics and Genomics and Association of Molecular Pathology [23] were used to evaluate the latter set of variants. The Genome Aggregation Database (gnomAD, accessed October 2017) [24] was used to identify a set of presumably benign, "control" variants (hereafter referred to as benign variants). All missense changes detected in males were selected $(n=$ 322 ). According to the gnomAD curation team every effort was made to exclude individuals with severe paediatric diseases from the dataset so we do not expect the overwhelming majority of these variants to be associated with iCSNB [24] [online: http://gnomad.broadinstitute.org/faq; accessed January 2019].

\section{Homology modelling}

A homology model of $\mathrm{Ca}_{\mathrm{v}} 1.4 \alpha 1$ was generated using MODELLER v9.17 [25], since its 3D structure has not been experimentally determined. The CACNAIF sequence from UniProt (Uniprot ID: O60840, [26]) was used to identify the structure of the rabbit $\mathrm{Ca}_{\mathrm{v}} 1.1$ complex from the Protein Data Bank (PDB, [27]) as a homologous structure (PDB ID: 5GJV). The sequences were aligned using Clustal Omega v1.2.3 [28] with default parameters. Approximately $64 \%$ sequence identity in the modelled regions suggests similarity in structure. Five models of $\mathrm{Ca}_{\mathrm{v}} 1.4 \alpha 1$ were built and the one with the lowest Discrete Optimised Protein Energy score was selected. PyMol [29] was used to visualise the model.

\section{Hypotheses and analyses}

To examine van der Waals interactions in the model, hydrogen atoms were added with Reduce [30], and atomic contacts were calculated with Probe [31]. Amino-acid replacements were modelled and visualised using KiNG v2.23 [32]. All low energy side chain conformations (rotamers) were examined and the one with smallest van der Waals overlaps chosen for each variant. In addition, the Richards volume scale [33] was used to calculate the differences in residue volumes. A reference set of all possible differences in volume of all 20 amino acids was calculated; the results of this were divided into four bins based on volume change upon amino-acid replacement: $<-42 \AA^{3}$ group, -42 to $0 \AA^{3}$ group, 0 to $42 \AA^{3}$ group and $>42 \AA^{3}$ group. Similarly, both the disease-implicated and the 
presumably benign set of variants were divided into four bins and were later compared with a replication the same four bins of variants with the only difference of having the mutant/introduced residues randomly generated using Monte Carlo simulation to identify statistically significant differences $(p<0.05)$.

The EGS hydrophobicity scale [34] was used to calculate change in hydrophobicity arising from an amino-acid replacement. To investigate changes in charged residues, hydrophobic residues, and differences in spatial distribution of the variants between the two sets, a reference set of uniformly distributed variants that were randomly generated using Monte Carlo simulation was used.

CACNA1F orthologues $(n=23)$ were identified from UniProt and aligned using Clustal Omega with default parameters. A conservation score was calculated through generating a profile [35] where the alignment is converted into a position-specific scoring system. The frequency with which the residues occur at each position is scored and later used to measure conservation using the substitution matrix BLOSUM62 [36]. The intracellular and extracellular sides of the plain in the model were determined by defining the spatial distribution of the residues using angle calculations. The angle formed between three residues (the $\mathrm{C} \alpha$ atom of the query residues, the centre of mass of the protein, and the $\mathrm{C} \alpha$ of Lys 383 chosen by inspection) was calculated. A residue was counted to be on the intracellular side of the plain if this angle was $\angle 90^{\circ}$, otherwise on the extracellular side of the plain. The carboxyl terminal domain (CTD) and the unmodelled parts were excluded from this calculation.

The scripts used in this study are available on the GitHub repository (https://github.com/shalawsallah/CACNA1F-va riants-analysis).

\section{Formulating pathogenicity criteria and evaluating prediction performance}

We defined two datasets. Dataset $\mathrm{D}$ represents diseaseimplicated variants from $\mathrm{HGMD}^{\mathrm{R}}$ and MGDL and dataset $\mathrm{N}$ represents presumably benign variants that were found in hemizygous state in the gnomAD cohort. We analysed these two datasets to find features that correlate with disease causality. The logistic regression algorithm "Logistic" [37] from the WEKA (Waikato Environment for Knowledge Analysis) machine learning package [38] was used with default parameters (weka.classifiers.functions.Logistic $-\mathrm{R}$ 1.0E-8 -M -1 -num-decimal-places 4) to classify the variants. Prior to this, the "ClassBalancer" and "Discretize" filters (weka.filters.MultiFilter -F "weka.filters.supervised. instance.ClassBalancer -num-intervals 10" -F "weka.filters. supervised.attribute.Discretize -R first-last -precision 6") were applied successively to reweight the imbalanced classes in the data and increase performance, respectively.
The performance of this classifier was later compared to that of the other four classifiers described above using the area under the curve (AUC) of receiver operating characteristic (ROC, [39]). The AUC under the precision recall (PR) curve was also used to measure their performances in correctly identifying the true positives, i.e. diseaseassociated variants, among the true positives and false positives [40]. The Matthews correlation coefficient test (MCC, [41]) was used to measure the correlation between the actual class of variants and the predictions made by the classifiers. The Bonferroni correction was applied to correct for possible error rates in multiple comparisons, such as comparisons made in domains of the protein [42].

\section{Results}

\section{CACNA1F variants identification}

We identified 72 disease-implicated missense variants (dataset D) that were present in either $\operatorname{HGMD}^{\mathrm{R}}(n=63)$ or the MGDL database $(n=9)$. Next, we identified 322 presumably benign missense variants (dataset $\mathrm{N}$ ) from gnomAD (the combined 394 variants are shown in Online Resource 1). Class assignment to datasets $\mathrm{D}$ and $\mathrm{N}$ was not definitive. Rather, it was assumed that the two groups of variants represented populations that were significantly skewed towards carrying disease-causing and benign variants, respectively.

\section{Performance of in silico tools}

We then assessed the ability of four in silico tools to predict the class of the CACNA1F missense variants (Table 1). The performance of these tools was highly variable, with a notable variation in the false positive rate (FPR). However, when using the unscaled/raw scores of CADD, at a threshold of 5.25, instead of the recommended scaled scores, we found it to perform better (e.g. $\mathrm{MCC}=0.53$, up from 0.12, AUC ROC $=0.83$, up from 0.79, and AUC $\mathrm{PR}=0.44$, up from 0.43). Furthermore, we found that changing the CONDEL-defined threshold from 0.52 to 0.65 ,

Table 1 The comparison of the true positive (TP) and false positive (FP) predictions of $C A C N A 1 F$ variants using four different tools (total positives and negatives $=72$ and 322, respectively (NM_005183.3; ENST00000376265.2)); FPR: false positive rate.

\begin{tabular}{lllll}
\hline Tools & Optimal threshold & TP & FP & FPR (\%) \\
\hline SIFT & 0.05 & 65 & 171 & 53 \\
PolyPhen2 & 0.85 & 62 & 132 & 41 \\
CADD & 15 & 70 & 277 & 86 \\
CONDEL & 0.52 & 69 & 255 & 79 \\
\hline
\end{tabular}




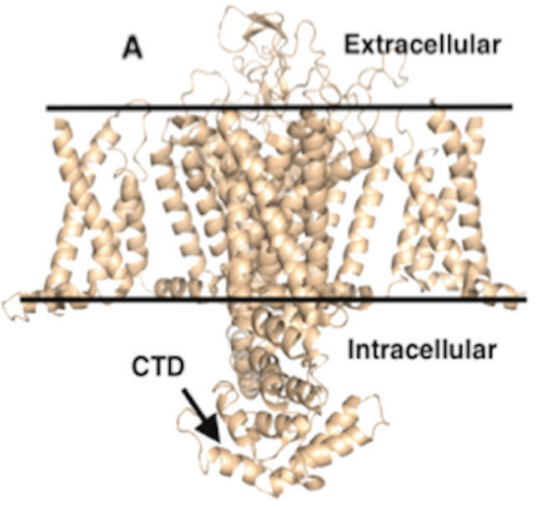

Fig. 1 Model representation of the template structure (PDB ID 5GJV) used in homology modelling. The structure is of the mammalian voltage-gated calcium channel $\mathrm{Ca}_{\mathrm{v}} 1.1$ complex at a resolution of 3.6 angstroms [43]. The transmembrane domain (approximately

i.e. a threshold specific to $C A C N A 1 F$, results in a higher overall performance (e.g. $\mathrm{MCC}=0.52$, up from 0.17 ).

\section{Homology modelling}

In order to analyse the structural and physicochemical properties of missense variants, we generated a homology model of $\mathrm{Ca}_{\mathrm{v}} 1.4 \alpha 1$ and mapped the variants onto it. Approximately $2 / 3$ of the human $\mathrm{Ca}_{\mathrm{v}} 1.4 \alpha 1$ protein could be modelled, i.e. residues $67-414,516-766$, and 858-1580. The parts that were not modelled had no homologous sequence in the template protein. Both termini of template $\mathrm{Ca}_{\mathrm{v}} 1.4 \alpha 1$ are on the cytoplasmic side and the structure has four (I-IV) transmembrane domains, each of which consists of six transmembrane $\alpha$-helical segments (S1-S6). The fourth transmembrane helix (S4) is a voltage sensor, with S5 and S6 segments of each domain making the ion channel and selectivity filter (Fig. 1, [43]).

\section{Structural analysis as a means of assessing variant pathogenicity}

In order to integrate clinical $C A C N A 1 F$ data with homology modelling of $\mathrm{Ca}_{\mathrm{v}} 1.4 \alpha 1$ we defined a set of structure-based parameters and determined their ability to differentiate variants from the $\mathrm{D}$ and $\mathrm{N}$ groups.

(i) The $C a_{v} 1.4 \alpha 1$ model. Four regions of the human $\mathrm{Ca}_{\mathrm{v}} 1.4 \alpha 1$ sequence, residues 1-66, 415-515, 767-857, and 1581-1977, have no homologous residues in the rabbit $\mathrm{Ca}_{\mathrm{v}} 1.1$ protein used as a template for modelling. The majority of the variants, i.e. $68 / 72$ (94\%), from dataset D were found to be on regions shared by both the model and the template structure, i.e. modelled regions, $(p<0.0001)$ compared with only $200 / 322(62 \%)$ of the variants from dataset $\mathrm{N}(p>0.9)$. The regions absent from the model, i.e. unmodelled regions, contain only a small proportion of the

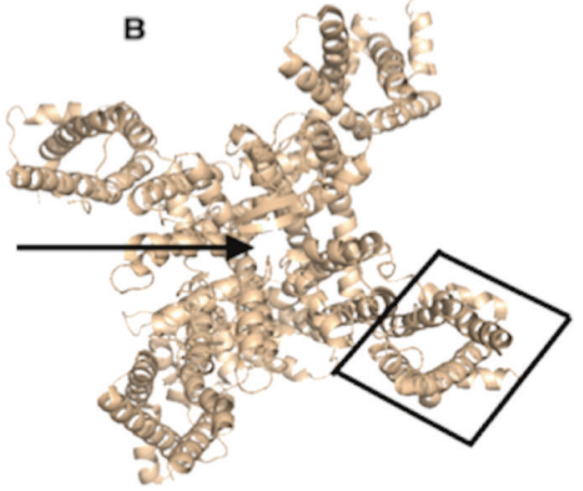

within the lines) in the side view representation (a). The pore (indicated by an arrow) and the first four out of six segments (highlighted in the rectangle) of each of the four domains in top view representation $(\mathbf{b})$.

variants from dataset $\mathrm{D}$ and are poorly conserved across ten human paralogues [43]. These data therefore suggest that variants found within the unmodelled regions are less likely to be disease-associated.

(ii) Variant location within the $C a_{v} 1.4 \alpha 1$ protein. Visual inspection suggested that the majority of the variants from dataset $\mathrm{D}$ were found closer to the intracellular region than to the extracellular one. We therefore defined a plain through the centre of mass of the molecule and determined whether variants were on the extracellular or intracellular side of this plain. This defined 745 residues to be in the extracellular side of the plain and 621 in the intracellular side of the plain. The locations of the variants differed between the two groups, with group D variants more frequently seen on the intracellular side of the plain of domain I $(p=0.048$ (a significant $p$ value must be $<0.005$ following Bonferroni correction)).

(iii) Conservation of mutated residues. Of the 72 mutated residues from dataset D, 69 were conserved among the 24 species considered (i.e. had a calculated conservation score $\leq 5$ out of 10). In contrast, of the 322 mutated residues from dataset $\mathrm{N}$ only 177 were conserved and 145 nonconserved (i.e. had a conservation score of $\geq 6$ out of 10 $\left(p=1.2 \times 10^{-11}\right.$, Mann-Whitney U test $\left.)\right)$.

When considering only the modelled regions, 67 of the 68 mutated residues from dataset D were established as conserved compared with 158 of the 200 mutated residues from dataset $\mathrm{N}\left(p=2.36 \times 10^{-25}\right.$, Mann-Whitney $\mathrm{U}$ test $)$. It can therefore be concluded that changes in conserved residues, the majority of which are on the modelled regions, are shown to be more likely disease-associated.

(iv) Changes in residue-volume and molecular goodness-of-fit test. Replacement of amino-acids may result in steric clashes with neighbouring regions of the protein model. To determine whether this is the case we assessed all low-energy side chain conformations [44] and evaluated 


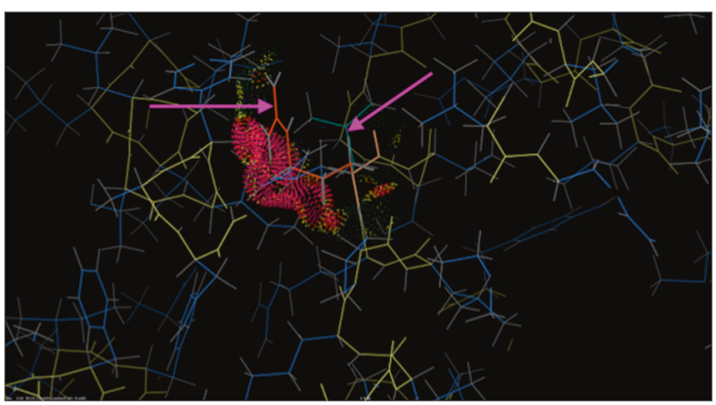

Fig. 2 Protein structure modelling for a disease-implicated variant. In testing the molecular goodness-of-fit for the disease-implicated CACNA1F (NM_005183.3; ENST00000376265.2) variant c.647 T > $\mathrm{G}$ p.(Leu216Arg), the red spikes reflect an overlap of van der Waals interaction between the surrounding residues and the introduced arginine (in orange) in place of the mutated leucine (in green) highlighted by the arrows.

their "goodness-of-fit" using the Probe software [31]. There is a significant difference between the two sets of data $(p=$ 0.001, Mann-Whitney U test) with a higher number of variants in dataset $\mathrm{D}$ having a more negative Probe score compared with the group $\mathrm{N}$ variants $(p=0.03$ at Probe scores <180, Mann-Whitney U test). This indicates the introduction of van der Waals overlaps resulting in steric clashes (Fig. 2).

The above finding is in accordance with the differences found in volume-change between the two groups of variants ( $p=0.03$, Mann-Whitney $\mathrm{U}$ test) with a higher number of variants in dataset $\mathrm{D}$ resulting in the replacement of smaller amino-acids with larger ones (i.e. a size change $<-42 \AA^{3}$, $n=23 / 72$ for dataset $\mathrm{D}, n=59 / 322$ for dataset $\mathrm{N} ; p=$ 0.046, Mann-Whitney U test). This difference was also observed when changes in volume in group $\mathrm{D}$ variants were compared with the reference set of volume changes $(p=$ 0.04, Mann-Whitney $\mathrm{U}$ test), in contrast to comparing the group $\mathrm{N}$ variants to the reference set $(p=0.50$, Mann-Whitney U test). Therefore, the changes that lead to disruption of packing, and a more negative Probe score are more likely to be disease-associated.

(v) Changes in charged residues. Cav $1.4 \alpha 1$ is a voltagegated calcium channel and alterations in charge are likely to affect its function. The replacement of neutral or negatively charged residues with positively charged residues, (gain of positive charge), was found to be more frequent among variants from dataset $\mathrm{D}, n=17 / 72$, than dataset $\mathrm{N}, n=34$ / 322 , throughout Cav1.4 $\alpha 1(p=0.036)$. There was more frequent replacement of positively charged residues with neutral or negatively charged residues (loss of positive charge) amongst variants from dataset $\mathrm{D}$ than dataset $\mathrm{N}$ in the fourth transmembrane helix (S4) (i.e. the voltage sensor) of all domains combined $(p=0.002), n=4 / 6$, and in S0-4 of domains II ( $p=0.01$ (significant $p$ value must be $<0.005$ following Bonferroni correction)), $n=2 / 4$, and in S0-4 of domain IV ( $p=0.002$ (significant $p$ value must be $<0.005$ following Bonferroni correction)), $n=3 / 5$.

(vi) Changes in hydrophobic residues. Since Cav1.4 $\alpha 1$ is a transmembrane protein, variants involving replacement of hydrophobic residues were considered. The replacement of hydrophobic residues among variants from dataset $\mathrm{D}$ in domain I, $n=12 / 16$, is correlated with pathogenicity ( $p=$ 0.015 (a significant $p$ value must be $<0.005$ following Bonferroni correction)).

\section{The pathogenicity criteria}

The pathogenicity criteria identified were used as features (Online Resource 1) by a logistic classifier to differentiate between the disease-implicated and presumably benign datasets as a composite assessment:

- Variants in sequences shared by the template structure and the model

- Loss of positively charged residues in the fourth transmembrane helix (S4) of the four homologous domains

- Gain of positively charged residues throughout the protein

- Loss of hydrophobic residues in domain I

- Variants at conserved residues

- Variants found in the lower half of domain I

- Variants resulting in the introduction of larger residues in place of smaller residues

- A more negative goodness-of-fit, i.e. Probe, score

\section{Machine learning application}

The logistic regression model "Logistic" from WEKA was used to classify the variants. The performance of the binary classifier is evaluated using a ROC curve [45] which measures trade-offs between the sensitivity and the specificity of the classifier at different thresholds. An optimum threshold can allow for a higher true positive rate (TPR) or a lower FPR, as required, or a combination of these in a diagnostic classifier. To account for the imbalance between the two classes in the data however, the ROC curve is combined with a PR curve to evaluate the true positives among the overall positive predictions.

The logistic model performance was compared with four commonly used in silico prediction tools (Table 2). Its performance in differentiating between the two classes of variants (AUC ROC $=0.84$ ) is comparable to that of the other four classifiers (Fig. 3). Notably, the larger area under the logistic model PR curve (AUC PR $=0.84$ ) represents a comparably high precision (representing a low FPR), and a 
Table 2 Comparing the predictions and the overall performance of the different tools shows a high recall rate at the expense of the precision rate at optimum thresholds for all the tools except for CACNA1F-vp. CACNA1F-vp has also a higher MCC score (MCC scores range from 1 to - 1 with 1 being a perfect correlation between predictions and the classes, and -1 being an inverse correlation); total disease-implicated and benign variants $=72$ and 322, respectively (NM_005183.3; ENST00000376265.2); AUC ROC: area under the receiver operating characteristic curve, $A U C P R$ : area under the precision recall curve, TPR: true positive rate, FPR: false positive rate, $P P V$ : positive predictive value, $M C C$ : Matthews Correlation Coefficient.

\begin{tabular}{llllllll}
\hline Tools & Threshold & Recall/TPR $(\%)$ & FPR $(\%)$ & Precision/PPV $(\%)$ & AUC ROC & AUC PR & MCC \\
\hline SIFT & 0.05 & 88 & 53 & 28 & 0.77 & 0.61 & 0.3 \\
PolyPhen2 & 0.85 & 86 & 41 & 32 & 0.83 & 0.59 \\
CADD & 15 & 97 & 86 & 20 & 0.79 & 0.43 \\
CONDEL & 0.522 & 96 & 79 & 21 & 0.85 & 0.12 \\
CACNA1F-vp & 0.567 & 86 & 33 & 72 & 0.84 & 0.61 \\
\hline
\end{tabular}

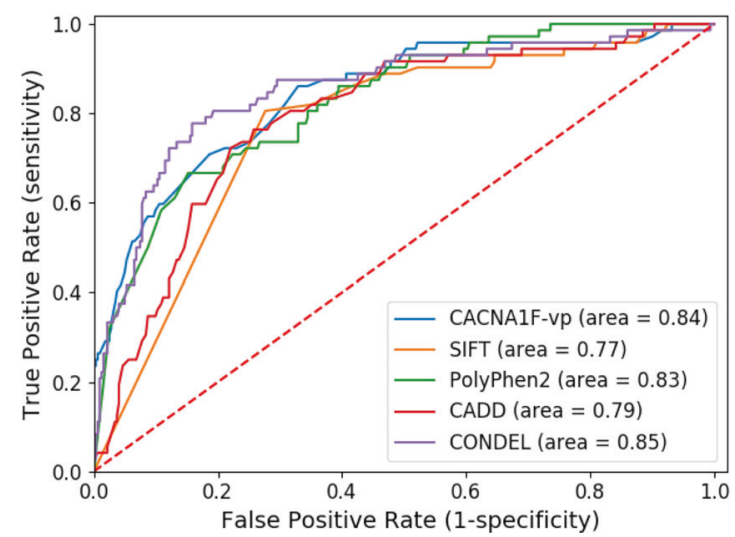

Fig. 3 ROC curves for the different classifiers. The predictive power of the protein-specific (CACNA1F-vp) model is comparable to that of the four tools, using 72 disease-implicated and 322 presumably benign $C A C N A 1 F$ variants, shown by an area under the receiver operating characteristic (ROC) curve of 0.84 .

high recall or sensitivity, i.e. a low false negative rate (Fig. 4).

\section{Discussion}

The interpretation of the large number of genetic variants generated through current gene sequencing techniques poses a significant challenge [46]. Computational prediction tools go some way to address this major issue but have been shown to frequently be inconsistent [20]. In this study, we produced a $C A C N A I F$-specific variant classifier through analysing sequence and structural data of the protein and its variants. This protein-specific approach was used as an alternative to currently available tools that tend to be less intuitive and often perform in a contradictory fashion $[47,48]$. Our analysis was enabled through the use of a 3D homology model of the protein structure that allowed structural analysis of $94 \%$ of the disease-implicated and $62 \%$ of the presumably benign variants. Clearly such analysis may not be possible for proteins where there is limited

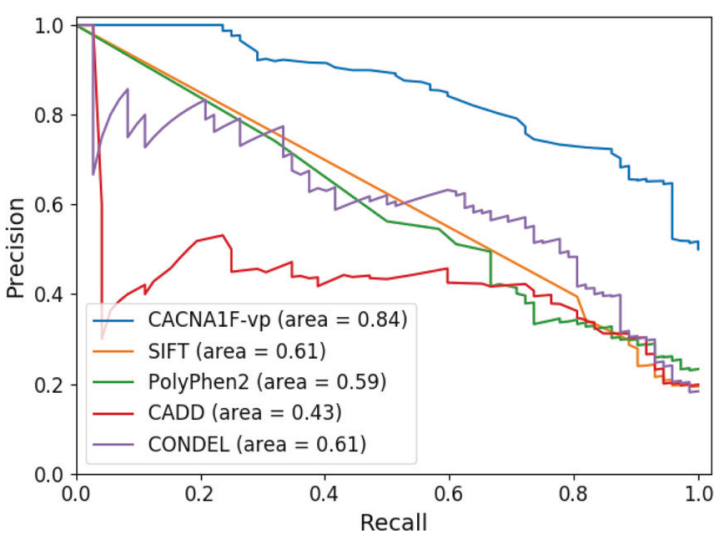

Fig. 4 PR curves for the different classifiers. The precision of the protein-specific (CACNA1F-vp) model is outperforming that of the four tools, using 72 disease-implicated and 322 presumably benign $C A C N A 1 F$ variants, shown by an area under the precision recall (PR) curve of 0.84

knowledge of the protein structure. Notably, the modelled region contained the majority of disease-implicated variants and appeared to be conserved among the orthologs of Cav1.4 $\alpha 1$. In contrast, the regions that were not included in the model contain only a small proportion of the diseaseimplicated variants and are poorly conserved across the orthologs and ten human paralogues of Cav1.4 $\alpha 1$. These results indicate a strong correlation firstly between the modelled protein regions and pathogenicity, and secondly between conservation and pathogenicity for this molecule.

The loss of positively charged residues in the fourth transmembrane helix (S4) found in the disease-implicated variant set, is thought to cause disturbance in voltagedependence functionality [49]. The outward movement of gating charges in S4 seem to result in bending of S6 and opening the pore [50]. Positively charged S4 residues form salt bridges with the negatively charged residues on S1-3 $[50,51]$; it can be speculated that altering these interactions affects channel structure and function. In contrast, diseaseimplicated variants leading to gain of positively charged residues may affect the overall function through interference 
with calcium ion selectivity and permeability [49]. In the CTD, such alterations may also interfere with inhibition of calcium dependent inactivation. Notably, this important regulatory functional domain tends to be less tolerant to variation [52].

We compared the prediction performances of four in silico tools to that of CACNA1F-vp, and found that the presented protein-specific model was specific and accurate. It could differentiate between disease-associated and benign variants as well as the other in silico tools (AUC ROC = 0.84; Table 2). Furthermore, our predictive model outperforms the other tools in correctly classifying the majority of the true disease-associated variants with a lower false positive prediction (AUC PR $=0.84$; Table 2). Including more presumably benign than disease-implicated variants in the analyses, i.e. having an imbalanced dataset, could improve the ROC curve without any real improvement in sensitivity or specificity. Evaluation using a PR curve is immune to this effect of an imbalanced dataset. This makes the PR curve a more robust measure to evaluate the specificity of each tool. CACNA1F-vp misclassified seven disease-implicated variants. These include the four diseaseimplicated variants found outside of the modelled regions of the protein (c.1301 C > T p.(Ala434Val), c. $1464 \mathrm{G}>\mathrm{T}$ p.(Glu488Asp), c.2390 A > T p.(Glu797Val), and c.2542 G $>$ A p.(Gly848Ser)), and one variant (c.1903G > A p.(Val635Ile)) that was seen in the gnomAD population at a high frequency (320/150041 alleles). The homology model was less informative for the variants found outside of the modelled regions. Hence, the lack of structural information about these variants may be a strong factor in their misclassification. CACNA1F-vp also misclassified 125/322 (39\%) benign variants (Online Resource 2). A recent study found that disease-implicated $C A C N A I F$ variants are present in gnomAD, which might be due to overlooked/ undiagnosed cases in this dataset [53]. When we used a more stringent criterion to define benign variants (presence in the gnomAD dataset in hemizygous state in at least five individuals) we found that the misclassification rate of CACNA1F-vp was lower $(16 / 52 ; 30 \%)$. Overall, we found significant differences between the CACNA1F-vp predictions and those of SIFT, CADD, and CONDEL $(p<$ 0.00001, McNemar chi square test [54]).

We found that adjusting the variant-pathogenicity thresholds defined by CADD and CONDEL improves the performance of these tools (MCC increases from 0.12 \& 0.17 to $0.53 \& 0.52$, respectively). Therefore, a proteinspecific pathogenicity-threshold in these tools further validates the advantage of using a protein-specific approach. A factor that could inflate the performance of these in silico methods is that the data used in testing these tools (in the present study) may have been utilised initially to train them. However probable, this was difficult to confirm.
Important insights could be gained by comparing the characteristics of the presumed disease-associated HGMD $^{\mathrm{R}}$, MGDL) and the presumed benign (gnomAD) variants. One of the key differences was in the molecular "goodness-of-fit" test where the deleterious packing interactions were shown to be greater amongst disease-implicated variants and are likely to lead to structural instability and functional abnormality. Intriguingly, a small number of the presumably benign variants $(n=19 / 322)$ were also found to have significantly disordered packing interactions (Online Resource 3); the majority of these changes (14/19) were among the CACNA1F-vp misclassified variants (Online Resource 2). A possible explanation for this is the inaccuracies in the homology modelling process around these missense changes. Alternatively, it is not implausible that some of these benign variants are in fact disease-associated (especially the extremely rare ones such as c.2221 C $>\mathrm{T}$ p.(Leu741Phe) which is found in $1 / 86168$ gnomAD alleles), Importantly, gnomAD is population rather than an unaffected control database and some individuals may in fact have iCSNB.

This study has important limitations. First, the missense changes that fall outside of the reliably modelled regions are more difficult to interpret using the approach outlined. This is highlighted by the misclassification of the four disease-implicated variants that fall outside of the modelled regions. Second, the protein-specific nature of our classifier has significant advantages but limits the number of available variants to train the model. Given the prevalence and degree of allelic heterogeneity of iCSNB, assembling a large independent variant dataset to test the classifier's performance was not possible. Third, we did not take into account factors like penetrance and expressivity in assembling the disease-implicated (dataset D) and presumably benign (dataset $\mathrm{N}$ ) variant datasets. It is worth noting though that, despite the fact that a number of different ophthalmic conditions has been linked to CACNA1F variants (including iCSNB, Åland eye disease and X-linked cone-rod dystrophy 3 ), incomplete penetrance is certainly not a frequent feature of $C A C N A I F$-related disorders [52, 55]. Intriguingly, eight disease-implicated variants were also present in gnomAD in hemizygous state (and where therefore also included in the presumably benign dataset; Online Resource 4). This highlights the fact that certain variants would have been incorrectly annotated; clearly, this lack of definitive variant class assignment negatively affects the performance of CACNA1F-vp.

We can conclude that CACNA1F-vp can form the basis of an effective test. Its relatively higher precision compared with existing tools may help pinpoint disease-associated variants among background variation, facilitating the process of diagnosing patients with iCSNB. Importantly, wrongly diagnosing affected individuals can cause distress to the patient and their family and can lead to further unnecessary 
investigations (for example repeated electrodiagnostic assessments). Obtaining co-segregation and functional data is undeniably important and necessary but this information is often difficult or impractical to get. We therefore believe that the presented classifier has a role in the evaluation of individuals with iCSNB. Finally, it can be speculated that through studying different molecules using similar approaches, a set of pathogenicity rules will emerge including protein-specific, family-specific or even perhaps more general rules.

Acknowledgements We would like to thank Prof Magnus Rattray and Dr Mudassar Iqbal from the division of Informatics, Imaging and Data Sciences at the University of Manchester for their guidance, and everyone else involved in this study. This work was supported by the Medical Research Council and Congenica Ltd.

Funding Medical Research Council (ref: 1790437).

\section{Compliance with ethical standards}

Conflict of interest Dr Lench is an employee of Congenica Ltd. All other authors declare that they have no conflict of interest.

Publisher's note Springer Nature remains neutral with regard to jurisdictional claims in published maps and institutional affiliations.

Open Access This article is licensed under a Creative Commons Attribution 4.0 International License, which permits use, sharing, adaptation, distribution and reproduction in any medium or format, as long as you give appropriate credit to the original author(s) and the source, provide a link to the Creative Commons license, and indicate if changes were made. The images or other third party material in this article are included in the article's Creative Commons license, unless indicated otherwise in a credit line to the material. If material is not included in the article's Creative Commons license and your intended use is not permitted by statutory regulation or exceeds the permitted use, you will need to obtain permission directly from the copyright holder. To view a copy of this license, visit http://creativecommons. org/licenses/by/4.0/.

\section{References}

1. MacArthur DG, Manolio TA, Dimmock DP, Rehm HL, Shendure $\mathrm{J}$, Abecasis GR, et al. Guidelines for investigating causality of sequence variants in human disease. Nature. 2014;508:469.

2. Taylor RL, Parry NRA, Barton SJ, Campbell C, Delaney CM, Ellingford JM, et al. Panel-based clinical genetic testing in 85 children with inherited retinal disease. Ophthalmology. 2017;124: 985-91.

3. Ellingford JM, Barton S, Bhaskar S, O'Sullivan J, Williams SG, Lamb JA, et al. Molecular findings from 537 individuals with inherited retinal disease. J Med Genet. 2016;53:761-7.

4. Sloan-Heggen CM, Bierer AO, Shearer AE, Kolbe DL, Nishimura $\mathrm{CJ}$, Frees KL, et al. Comprehensive genetic testing in the clinical evaluation of 1119 patients with hearing loss. Hum Genet. 2016;135:441-50.

5. Cooper GM, Shendure J. Needles in stacks of needles: finding disease-causal variants in a wealth of genomic data. Nat Rev Genet. 2011;12:628-40.
6. Astuti GDN, van den Born LI, Khan MI, Hamel CP, Bocquet B, Manes G, et al. Identification of inherited retinal diseaseassociated genetic variants in 11 candidate genes. Genes. 2018;9.

7. Liew G, Michaelides M, Bunce C. A comparison of the causes of blindness certifications in England and Wales in working age adults (16-64 years), 1999-2000 with 2009-2010. BMJ Open. 2014;4:e04015.

8. Zeitz C, Robson AG, Audo I. Congenital stationary night blindness: an analysis and update of genotype-phenotype correlations and pathogenic mechanisms. Prog Retinal Eye Res. 2015;45 Suppl C:58-110.

9. Bech-Hansen NT, Naylor MJ, Maybaum TA, Pearce WG, Koop B, Fishman GA, et al. Loss-of-function mutations in a calciumchannel I[alpha]|1-subunitgene in Xp11.23 cause incomplete Xlinked congenital stationary night blindness. Nat Genet. 1998;19: 264-7.

10. Strom TM, Nyakatura G, Apfelstedt-Sylla E, Hellebrand H, Lorenz B, Weber BH, et al. An L-type calcium-channel gene mutated in incomplete $\mathrm{X}$-linked congenital stationary night blindness. Nat Genet. 1998;19:260-3.

11. Striessnig J, Hoda JC, Koschak A, Zaghetto F, Mullner C, Sinnegger-Brauns $\mathrm{MJ}$, et al. L-type $\mathrm{Ca} 2+$ channels in $\mathrm{Ca} 2+$ channelopathies. Biochem Biophys Res Commun. 2004;322: 1341-6.

12. Stenson PD, Mort M, Ball EV, Evans K, Hayden M, Heywood S, et al. The human gene mutation database: towards a comprehensive repository of inherited mutation data for medical research, genetic diagnosis and next-generation sequencing studies. Hum Genet. 2017;136:665-77.

13. Ng PC, Henikoff S. SIFT: predicting amino acid changes that affect protein function. Nucleic Acids Res. 2003;31:3812-4.

14. Adzhubei IA, Schmidt S, Peshkin L, Ramensky VE, Gerasimova A, Bork P, et al. A method and server for predicting damaging missense mutations. Nat Methods. 2010;7:248-9.

15. Kircher M, Witten DM, Jain P, O'Roak BJ, Cooper GM, Shendure J. A general framework for estimating the relative pathogenicity of human genetic variants. Nat Genet. 2014;46:310.

16. González-Pérez A, López-Bigas N. Improving the assessment of the outcome of nonsynonymous SNVs with a consensus deleteriousness score, condel. Am J Hum Genet. 2011;88:440-9.

17. Pires AS, Porto WF, Franco OL, Alencar SA. In silico analyses of deleterious missense SNPs of human apolipoprotein E3. Sci Rep. 2017;7:2509.

18. Reva B, Antipin Y, Sander C. Predicting the functional impact of protein mutations: application to cancer genomics. Nucleic Acids Res. 2011;39:e118.

19. Shihab HA, Gough J, Cooper DN, Stenson PD, Barker GL, Edwards KJ, et al. Predicting the functional, molecular, and phenotypic consequences of amino acid substitutions using hidden Markov models. Hum Mutat. 2013;34:57-65.

20. Chun, S. and J. C. Fay. "Identification of deleterious mutations within three human genomes." Genome Research. 2009;19: 1553-61

21. Leong IU, Stuckey A, Lai D, Skinner JR, Love DR. Assessment of the predictive accuracy of five in silico prediction tools, alone or in combination, and two metaservers to classify long QT syndrome gene mutations. BMC Med Genet. 2015;16:34.

22. Landrum MJ, Lee JM, Benson M, Brown G, Chao C, Chitipiralla $\mathrm{S}$, et al. ClinVar: public archive of interpretations of clinically relevant variants. Nucleic Acids Res. 2016;44:D862-8.

23. Richards S, Aziz N, Bale S, Bick D, Das S, Gastier-Foster J, et al. Standards and guidelines for the interpretation of sequence variants: a joint consensus recommendation of the American College of Medical Genetics and Genomics and the Association for Molecular Pathology. Genet Med. 2015;17:405-24. 
24. Karczewski KJ, Francioli LC, Tiao G, Cummings BB, Alföldi J, Wang Q, et al. Variation across 141,456 human exomes and genomes reveals the spectrum of loss-of-function intolerance across human protein-coding genes. 2019. bioRxiv: 531210 .

25. Webb B, Sali A. Comparative protein structure modeling using Modeller. Curr Protoc Bioinforma. 2016;54:5.6.1-5.6.37.

26. Bateman A, O'Donovan C, Magrane M, Alpi E, Antunes R, Bely B, et al. UniProt: the universal protein knowledgebase. Nucleic Acids Res. 2018;45:D158-69.

27. Berman HM, Westbrook J, Feng Z, Gilliland G, Bhat TN, Weissig $\mathrm{H}$, et al. The protein data bank. Nucleic Acids Res. 2000;28: 235-42.

28. Sievers F, Wilm A, Dineen D, Gibson TJ, Karplus K, Li W, et al. Fast, scalable generation of high-quality protein multiple sequence alignments using clustal omega. Mol Syst Biol. 2011;7:539.

29. Schrodinger LLC. The PyMOL molecular graphics system. Version. 2015;1:8.

30. Word JM, Lovell SC, Richardson JS, Richardson DC. Asparagine and glutamine: using hydrogen atom contacts in the choice of side-chain amide orientation. J Mol Biol. 1999;285: 1735-47.

31. Word JM, Lovell SC, LaBean TH, Taylor HC, Zalis ME, Presley BK, et al. Visualizing and quantifying molecular goodness-of-fit: small-probe contact dots with explicit hydrogen atoms. J Mol Biol. 1999;285:1711-33.

32. Chen VB, Davis IW, Richardson DC. KING (Kinemage, next generation): a versatile interactive molecular and scientific visualization program. Protein Sci. 2009;18:2403-9.

33. Richards FM. Areas, Volumes, packing, and protein structure. http://dxdoiorg/101146/annurevbb06060177001055. 1977.

34. Engelman DM, Steitz TA, Goldman A. Identifying nonpolar transbilayer helices in amino acid sequences of membrane proteins. http://dxdoiorg/101146/annurevbb15060186001541. 1986.

35. Stevens TA. Python programming for biology, bioinformatics, and beyond. Boucher WA, editor: Cambridge: Cambridge University Press; 2015.

36. Henikoff S, Henikoff JG. Amino acid substitution matrices from protein blocks. Proc Natl Acad Sci USA. 1992;89:10915-9.

37. Le Cessie SVH, Ridge JC. Estimators in logistic regression. J R Stat Soc Ser C (Appl Stat). 1992;41:11.

38. Witten IH, Frank E, Hall MA, Pal CJ. Data mining, Fourth edition: Practical Machine Learning Tools and Techniques: Morgan Kaufmann Publishers Inc.; 2016. 654 p.

39. DeLong ER, DeLong DM, Clarke-Pearson DL. Comparing the areas under two or more correlated receiver operating characteristic curves: a nonparametric approach. Biometrics. 1988;44:837-45.
40. Saito T, Rehmsmeier M. The precision-recall plot is more informative than the ROC plot when evaluating binary classifiers on imbalanced datasets. PLoS ONE. 2015;10:e0118432.

41. Matthews BW. Comparison of the predicted and observed secondary structure of T4 phage lysozyme. Biochim Biophys Acta. 1975;405:442-51.

42. Dunn OJ. Multiple comparisons among means. J Am Stat Assoc. 1961;56:52-64.

43. Wu J, Yan Z, Li Z, Qian X, Lu S, Dong M, et al. Structure of the voltage-gated calcium channel $\mathrm{Ca}(\mathrm{v}) 1.1$ at $3.6 \mathrm{~A}$ resolution. Nature. 2016;537:191-6.

44. Lovell SC, Word JM, Richardson JS, Richardson DC. The penultimate rotamer library. Proteins. 2000;40:389-408.

45. Hajian-Tilaki K. Receiver operating characteristic (ROC) curve analysis for medical diagnostic test evaluation. Casp J Intern Med. 2013;4:627-35.

46. Lappalainen T, Scott AJ, Brandt M, Hall IM. Genomic analysis in the age of human genome sequencing. Cell. 2019;177:70-84.

47. Williams S. Analysis of in silico tools for evaluating missense variants. National Genetics Reference Laboratory (Manchester). 2012.

48. de la Campa E, Padilla N, de la Cruz X Development of pathogenicity predictors specific for variants that do not comply with clinical guidelines for the use of computational evidence. BMC Genomics. 2017;18(Suppl 5):569.

49. Hess EJ. Migraines in mice? Cell. 1996;87:1149-51.

50. Catterall WA. Ion channel voltage sensors: structure, function, and pathophysiology. Neuron. 2010;67:915-28.

51. Striessnig J, Bolz HJ, Koschak A. Channelopathies in Cav1.1, Cav1.3, and Cav1.4 voltage-gated L-type Ca2 + channels. Pflug Arch. 2010;460:361-74.

52. Zeitz C, Robson AG, Audo I. Congenital stationary night blindness: an analysis and update of genotype-phenotype correlations and pathogenic mechanisms. Prog Retin Eye Res. 2015;45:58-110.

53. Zeitz C, Michiels C, Neuille M, Friedburg C, Condroyer C, Boyard $\mathrm{F}$, et al. Where are the missing gene defects in inherited retinal disorders? intronic and synonymous variants contribute at least to $4 \%$ of CACNA1F-mediated inherited retinal disorders. Hum Mutat. 2019;40:765-87.

54. McNemar Q. Note on the sampling error of the difference between correlated proportions or percentages. Psychometrika. 1947;12: $153-7$.

55. Hove MN, Kilic-Biyik KZ, Trotter A, Grønskov K, Sander B, Larsen $\mathrm{M}$, et al. Clinical characteristics, mutation spectrum, and prevalence of Åland eye disease/incomplete congenital stationary night blindness in Denmark. Invest Ophthalmol Vis Sci. 2016;57:6861-9. 